\title{
Quercetin protects rat cortical neurons against traumatic brain injury
}

\author{
GUOLIANG DU ${ }^{1}$, ZONGMAO ZHAO ${ }^{2}$, YONGHAN CHEN $^{1}$, ZONGHAO LI $^{1}$, \\ YAOHUI TIAN $^{1}$, ZHIFENG LIU ${ }^{1}$, BIN LIU ${ }^{1}$ and JIANQIANG SONG ${ }^{1}$ \\ ${ }^{1}$ Department of Neurosurgery, Central Hospital of Cangzhou, Cangzhou, Hebei 061001; ${ }^{2}$ Department of Neurosurgery, \\ Second Hospital of Hebei Medical University, Shijiazhuang, Hebei 060000, P.R. China
}

Received October 24, 2016; Accepted June 8, 2017

DOI: $10.3892 / \mathrm{mmr} .2018 .8801$

\begin{abstract}
Previous studies have demonstrated that traumatic brain injury (TBI) may cause neurological deficits and neuronal cell apoptosis. Quercetin, one of the most widely distributed flavonoids, possesses anti-inflammatory, anti-blood coagulation, anti-ischemic and anti-cancer activities, and neuroprotective effects in the context of brain injury. The purpose of the present study was to investigate the neuroprotective effects of quercetin in TBI. A total of 75 rats were randomly arranged into 3 groups as follows: Sham group (Sham); TBI group (TBI); and TBI + quercetin group (Que). Brain edema was evaluated by analysis of brain water content. The neurobehavioral status of the rats was evaluated by Neurological Severity Scoring. Immunohistochemical and western blot analyses were used to measure the expression of certain proteins. The results of the present study demonstrated that post-TBI administration of quercetin may attenuate brain edema, in addition to improving motor function in rats. Additionally, quercetin caused a marked inhibition of extracellular signal-regulated kinase $1 / 2$ phosphorylation and activated Akt serine/threonine protein kinase phosphorylation, which may result in attenuation of neuronal apoptosis. The present study provided novel insights into the mechanism through which quercetin may exert its neuroprotective activity in a rat model of TBI.
\end{abstract}

Correspondence to: Professor Guoliang Du, Department of Neurosurgery, Central Hospital of Cangzhou, 201 Xinhua Road, Cangzhou, Hebei 061001, P.R. China

E-mail: guoliangdu2001@163.com

Abbreviations: TBI, traumatic brain injury; NSS, neurological severity score; Que, quercetin-treated; WW, wet weight; DW, dry weight; $\mathrm{H} \& \mathrm{E}$, hematoxylin and eosin; PI3K, phosphatidylinositol 3-kinase; Akt, Akt serine/threonine protein kinase; ERK, extracellular signal-regulated kinase

Key words: quercetin, apoptosis, phosphatidylinositol 3-kinase PI3K/Akt, extracellular signal-regulated kinase $1 / 2$, traumatic brain injury

\section{Introduction}

Traumatic brain injury (TBI) is a leading cause of mortality and morbidity worldwide (1). TBI patients suffer permanent neurological and psychological disabilities that represent a significant social and economic burden. TBI-induced deficits are due to primary (mechanical impact) and secondary (delayed) injuries. It is essential to elucidate the biological cascades that drive the delayed secondary phase subsequent to TBI (2). Despite considerable progress being made in animal models and preclinical research in recent years, there are currently no available therapeutic strategies in clinical practice for TBI.

The flavonoid quercetin $(3,5,7,30,40$-pentahydroxyflavone), one of the most widely distributed flavonoids in fruits and vegetables, is known to be a potent anti-oxidant and free radical scavenger (3). A number of studies have demonstrated that quercetin possesses anti-inflammatory, anti-coagulation, anti-ischemic and anti-cancer activities (4-6). In addition, Yang et al (7) suggested that in TBI rats, quercetin improves cognitive function owing to its neuroprotective action via the inhibition of oxidative stress, leading to a reduced inflammatory response, thereby reducing neuronal death. It was hypothesized that post-injury treatment with quercetin may exert a therapeutic effect against TBI. Our previous study (8) provided results similar to Yang, however, the specific molecular mechanisms requires further study. The present study also analyzed the expression of Akt serine/threonine protein kinase, phosphorylated (p)-Akt, extracellular signal-regulated kinase $(E R K) 1 / 2$ and p-ERK1/2 in injured neurons in the cortex, which serves an important role in signal transduction following TBI. The aim of the present study was to investigate the protective effects of quercetin on neurological impairment and spatial cognitive function after TBI in rat model. It further examined whether quercetin could attenuate neuronal apoptosis via PI3K/Akt and ERK1/2 signaling, thereby reducing brain damage.

\section{Materials and methods}

Animals. The Institutional Animal Care and Use Committee of Hebei Medical University (Shijiazhuang, China) approved all experiments, which were performed according to the 
guidelines of the National Institutes of Health (NIH) Guide for the Care and Use of Laboratory Animals (NIH Publications no. 80-23, revised 1978; NIH, Bethesda, MD, USA). All efforts were made to minimize the number of animals used and their suffering. A total of 75 male Sprague Dawley rats, weighing 280-320 g (6-8 weeks), were supplied from the Experimental Animal Center of Hebei Medical University (Shijiazhuang, Hebei, China). All animals were housed in plastic boxes at a temperature of $22-24^{\circ} \mathrm{C}, 50 \%$ humidity and were provided food and water ad libitum under a 12-h reversed light-dark cycle.

Model of TBI. The TBI model was produced using a modified weight-drop device (9). Following $10 \%$ chloral hydrate anesthesia $(3 \mathrm{ml} / \mathrm{kg})$, a midline longitudinal incision was performed to expose the skull between bregma and lambda suture lines. A steel disk (diameter, $10 \mathrm{~mm}$; thickness, $3 \mathrm{~mm}$ ) was adhered to the skull using dental acrylic. Animals were moved onto a foam mattress underneath a weight-drop device where a weight of $450 \mathrm{~g}$ fell freely through a vertical tube from $1.5 \mathrm{~m}$ onto the steel disk. Sham-operated animals underwent the same surgical procedure without weight-drop impact. Rats were placed on heat pads $\left(37^{\circ} \mathrm{C}\right)$ for $2-4 \mathrm{~h}$ to maintain normal body temperature during the recovery period.

Groups and drug administration. All rats were randomly arranged into 3 groups as follows: Sham group $(n=25)$; TBI group $(n=25)$; and TBI + quercetin group (Que; $n=25)$. Quercetin (Sigma-Aldrich; Merck KGaA, Darmstadt, Germany; dissolved in $0.9 \%$ saline solution) was administered intraperitoneally at a dose of $50 \mathrm{mg} / \mathrm{kg}$ at $30 \mathrm{~min}, 12$ and $24 \mathrm{~h}$ following the TBI insult.

In addition, 15 rats (5/group) underwent behavioral testing. All investigations were blinded and the animal groupings were revealed only at the end of the behavioral and histological analyses.

Evaluation of brain edema. Brain water content was determined at day 1, 3 and 5 following TBI (45 rats, 15/group). In order to reduce the use of animal population, at 5 days after TBI, 15 rat brains were taken from rats which had completed the behavioral experiments. Rat brains were separated and weighed immediately with a chemical balance to obtain the wet weight $(\mathrm{WW})$. Following drying in a desiccating oven for $24 \mathrm{~h}$ at $100^{\circ} \mathrm{C}$, dry tissues were weighed again to obtain the constant dry weight (DW). The percentage of water in the tissues was calculated according to the following formula: brain water $(\%)=[(\mathrm{WW}-\mathrm{DW}) / \mathrm{WW}] \times 100$.

Recovery of motor function. The neurobehavioral status of the rats was evaluated at day 1,3 and 5 after TBI using a set of 10 tasks, collectively termed the Neurological Severity Score (NSS) (10), which test reflexes, alertness, coordination and motor abilities. A point is awarded for failure to perform a particular task; therefore, a score of 10 reflects maximal impairment, whereas a healthy rat scores 0. Post-injury, NSS was evaluated at day 1, 3 and 5. Each animal was assessed by an observer who was blinded to the treatment group of the animal. The difference between the initial NSS (performed at day 1) and that at any subsequent time point was calculated for each rat, and this value ( $\triangle \mathrm{NSS}$ ) reflects the spontaneous or treatment-induced recovery of motor function.

Hematoxylin and eosin $(H \& E)$ staining and neuron count. At $24 \mathrm{~h}$ post-TBI, 15 rats (5/group) were anesthetized as described above, and perfused intracardially with isotonic sodium chloride solution, followed by $4 \%$ (w/v) paraformaldehyde in $0.1 \mathrm{M}$ sodium phosphate buffer $(\mathrm{pH}=7.4)$. The brains were removed and fixed for $48 \mathrm{~h}$ in $4 \%$ (w/v) paraformaldehyde at $22-24^{\circ} \mathrm{C}$. Following fixation, brains were embedded in paraffin, and sliced into $6 \mu \mathrm{m}$ coronal sections at the level of the bregma and stained with hematoxylin (20 min) and eosin $(3 \mathrm{sec})$ at $22-24^{\circ} \mathrm{C}$. The staining was visualized by light microscopy at x400 magnification (Olympus Corporation, Tokyo, Japan). The surviving and dying neurons per $\mathrm{mm}^{2}$ cortex were quantified (the nuclei of dead cells were shrunk and thickened).

Immunohistochemical. As for HE staining, the brain tissues were fixed, embedded and cut into $6 \mu \mathrm{m}$ slices. Sections were deparaffinized with xylene and rehydrated at $60^{\circ} \mathrm{C}$ with graded ethanol (100, 95, 90, 80 and 70\%). Endogenous peroxidase activity was blocked using $3 \%$ hydrogen peroxide for $30 \mathrm{~min}$ at room temperature, followed by $5 \%$ normal goat serum (AR0009; Wuhan Boster Biological Technology, Ltd., Wuhan, China) for $1 \mathrm{~h}$ to block non-specific protein interactions. The sections were subsequently incubated overnight at $4^{\circ} \mathrm{C}$ with an anti-activated caspase 3 antibody (1:500; AB2302; Abcam, Cambridge, UK). Following three washes with PBS, the slides were incubated with a biotinylated goat anti-mouse horseradish peroxidase conjugated secondary antibody (1:100, BA1051; Wuhan Boster Biological Technology Ltd.) for $2 \mathrm{~h}$ at room temperature. The sections were washed with PBS again, and incubated with the kit-provided horseradish peroxidase (HRP)-streptavidin for $30 \mathrm{~min}$ at room temperature. The peroxidase reaction was visualized using $0.05 \%$ diaminobenzidine $+0.01 \%$ hydrogen peroxide. Immunohistochemical procedures were performed in accordance with the manufacturer's protocols. The positive cells were visualized by a microscope at x100 magnification.

Western blotting. The rats were deeply anesthetized as described above $24 \mathrm{~h}$ following TBI. The cortical region of the rat brain was rapidly isolated. The segments were immediately stored at $-80^{\circ} \mathrm{C}$ for further analysis. Total protein samples were extracted from brain tissues using whole cell lysis buffer (WD2072; Beyotime Institute of Biotechnology, Shanghai, China) a bicinchoninic acid protein assay kit (P10310; Beyotime Institute of Biotechnology) was used to determine the protein concentration of each sample. The homogenate was heated to $100^{\circ} \mathrm{C}$ for $10 \mathrm{~min}$ and centrifuged again at $15,294 \mathrm{x} \mathrm{g}$ for $1 \mathrm{~min}$ at $22-24^{\circ} \mathrm{C}$. Equal amounts $(80 \mu \mathrm{g})$ of protein were subjected to Tris-HCl SDS-PAGE on 8 and $12 \%$ gels (Bio-Rad Laboratories, Inc., Hercules, CA, USA) for $30 \mathrm{~min}$ at $70 \mathrm{~V}$ and $60 \mathrm{~min}$ at $120 \mathrm{~V}$. Following electrophoresis, the proteins were transferred onto polyvinylidene fluoride membranes (EMD Millipore, Billerica, MA, USA) at $300 \mathrm{~mA}$ for immunoblotting. Following blocking with $5 \%$ skimmed milk for $2 \mathrm{~h}$ at room temperature, 
membranes were incubated overnight at $4^{\circ} \mathrm{C}$ with the following primary antibodies: Rabbit anti-activated Caspase 3 (AB2302; 1:1,000; Abcam), rabbit anti-Akt (AB81283; 1:1,000; Abcam), rabbit anti-p-Akt (AB38449; 1:1,000; Abcam), rabbit anti-ERK1/2 (AB17942; 1:1,000; Abcam), rabbit anti-p-ERK1/2 (AB214362; 1:1,000; Abcam), and rabbit anti- $\beta$-actin (AB227387; $1: 5,000 ;$ Abcam). Following three washes in TBS-Tween 20, membranes were incubated with a horseradish peroxidase-conjugated goat anti-rabbit immunoglobulin G (BL003A; 1:5,000; EMD Millipore) for $2 \mathrm{~h}$ at room temperature. Protein bands were visualized using an enhanced chemiluminescence kit (Beyotime Institute of Biotechnology). Band density was quantified via detection with a DNR Micro Chemi chemiluminescence gel imaging system (DNR Bio-Imaging Systems Ltd., Neve Yamin, Israel). Each band density was normalized to the density of $\beta$-actin.

Statistical analysis. SPSS software version 16.0 (SPSS, Inc., Chicago, IL, USA) was used for statistical analysis. A statistical evaluation of the data was performed using a one-way analysis of variance, followed by post hoc comparisons using the least significant difference or Kruskal-Wallis method. All experimental data are expressed as the mean \pm standard error of the mean, and $\mathrm{P}<0.05$ was considered to indicate a statistically significant difference.

\section{Results}

Quercetin attenuates TBI-induced cerebral edema. Following injury, brain edema leads to an elevation in intracranial pressure, reducing cerebral perfusion pressure and brain oxygenation. Edema is associated with the resultant pathology following TBI (11). In order to evaluate the effects of quercetin on brain edema, the wet-dry weight method was used in the present study to evaluate brain edema at day 1, 3 and 5 after TBI. As presented in Fig. 1, cerebral water content was significantly increased at day 1, 3 and 5 after TBI compared with the sham group $(\mathrm{P}<0.01)$. However, treatment with quercetin attenuated this increased compared with the TBI model group $(\mathrm{P}<0.05)$.

Quercetin attenuates TBI-induced motor deficits. Motor deficit recovery was expressed as $\triangle \mathrm{NSS}$ in present study. Alterations in the functional recovery of rats at day 1,3 and 5 are depicted in Fig. 2. It was observed that rats exhibited marked motor deficits following TBI. Post-injury administration of quercetin significantly improved the motor function between day 1 and 5 after trauma compared with the TBI group $(\mathrm{P}<0.05)$.

Quercetin increases neuronal survival in the cortical region of brain. Cortical regions of brains were collected and neuronal survival was assessed at $24 \mathrm{~h}$ via H\&E staining. As presented in Fig. 3, the nuclei of normal neurons were round and stained pale, whereas nuclei of dying neurons were pyknotic and darkly stained following TBI. The survival rate of neurons in the quercetin-treated group was significantly improved compared with that of the TBI group $(\mathrm{P}<0.05)$.

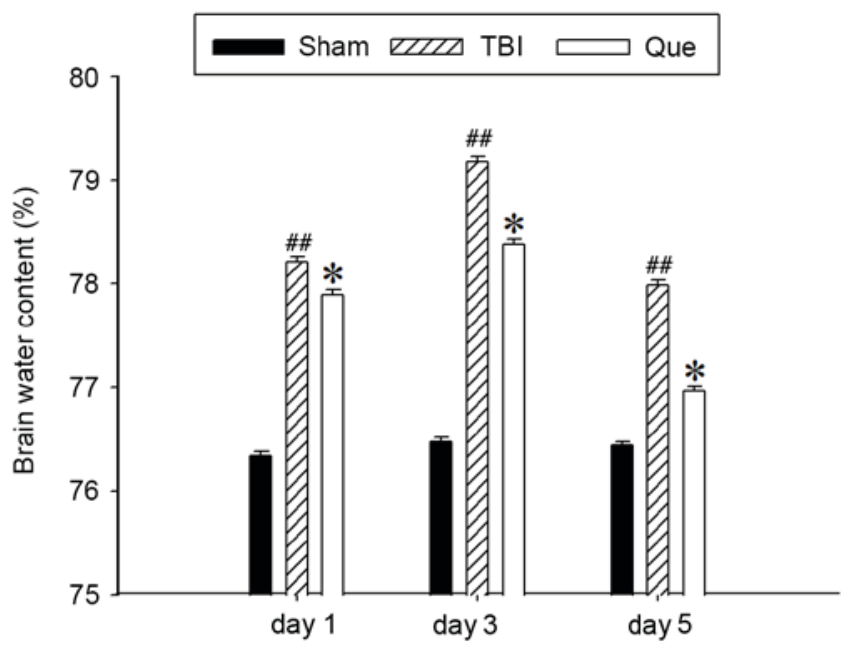

Figure 1. Effect of treatment with quercetin on brain edema. Brain water content was determined at day 1, 3 and 5 following TBI. Bars represent mean \pm standard error of the mean ( $n=5 /$ group). Cerebral water content was significantly increased at day 1,3 and 5 following TBI $\left({ }^{\#} \mathrm{P}<0.01\right.$ vs. sham group). However, treatment with quercetin attenuated this increase compared with the TBI model group ( $\mathrm{P}<0.05 \mathrm{vs}$. TBI group). TBI, traumatic brain injury; Que, quercetin-treated.

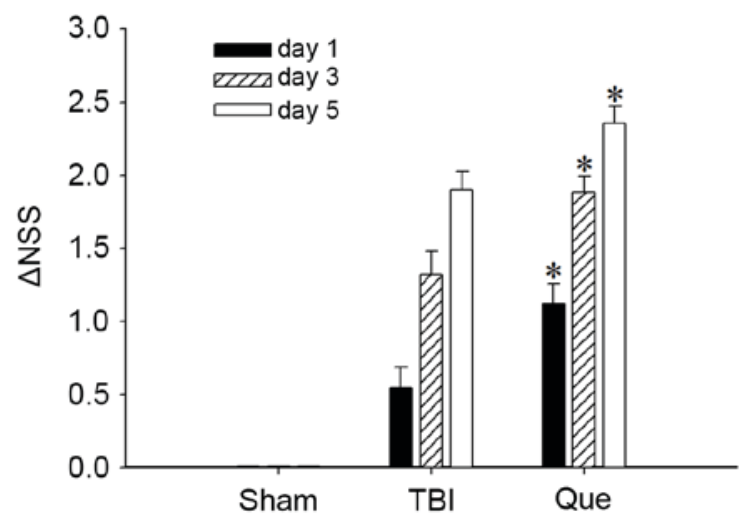

Figure 2. Effect of treatment with quercetin on TBI-induced motor deficits The temporal alterations in motor recovery were determined at day 1, 3 and 5 following TBI and calculated as $\triangle$ NSS. Bars represent mean \pm standard error of the mean ( $n=5 /$ group). Rats exhibited significant motor deficits post-TBI. Post-injury administration of quercetin significantly improved the motor function at day 1,3 and 5 following injury $\left({ }^{*} \mathrm{P}<0.05\right.$ vs. TBI group). TBI, traumatic brain injury; NSS, Neurological Severity Score; Que, quercetin-treated.

Quercetin attenuates neuronal apoptosis in the cortex. In order to assess the effect of quercetin on neuronal apoptosis following TBI, immunohistochemical and western blot analyses were used to assess alterations in caspase 3 expression, respectively. As depicted in Fig. 4A, representative photomicrographs exhibited a high density of caspase3-positive cells in the TBI group compared with the sham group at $24 \mathrm{~h}$. However, the expression of activated caspase 3 -positive cells notably decreased in the quercetin treatment group. In addition, western blot analysis revealed that, compared with the sham group, the protein expression levels of activated caspase 3 increased significantly in the TBI group at $24 \mathrm{~h}(\mathrm{P}<0.01)$, and the levels of caspase 3 exhibited a significant downregulation at the same time point following treatment with quercetin $(\mathrm{P}<0.05$; Fig. 4B and $\mathrm{C})$. 

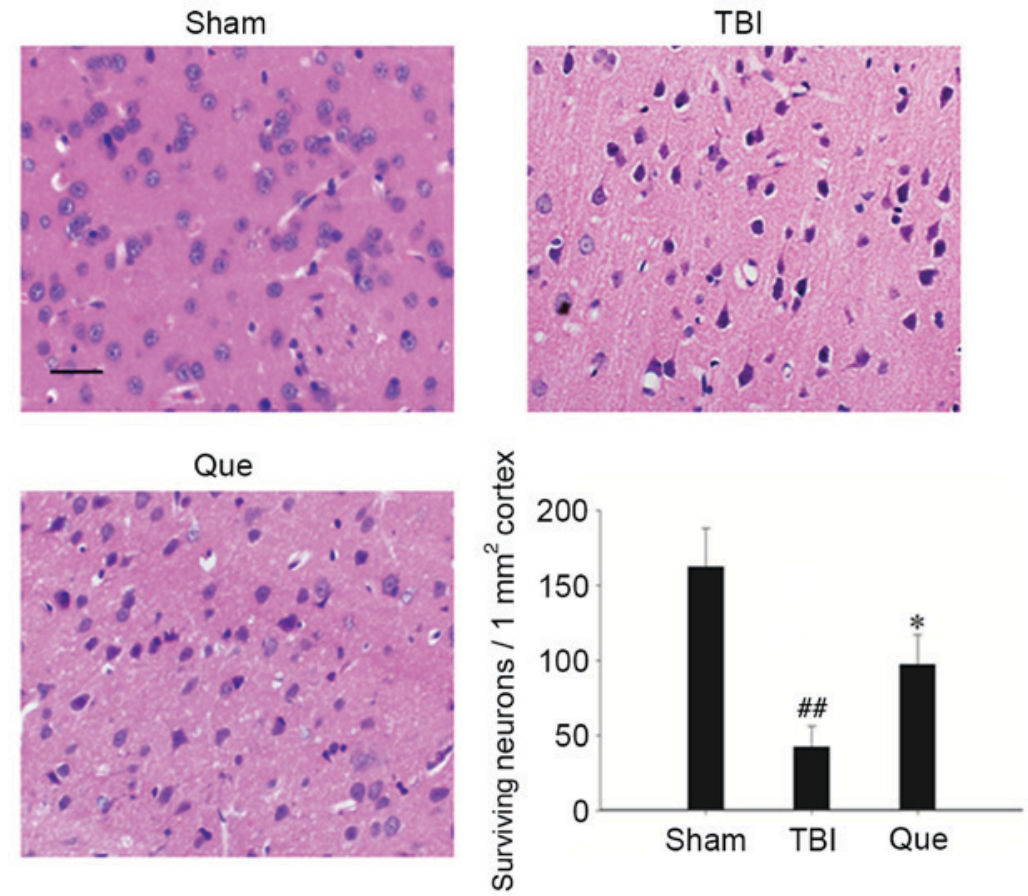

Figure 3. Effect of quercetin on the survival rates of neurons in the cortical region at $24 \mathrm{~h}$. Representative hematoxylin and eosin staining of neurons from the Sham, TBI and Que groups. Bars represent the percentages of surviving neurons as the mean \pm standard error of the mean ( $\mathrm{n}=5 / \mathrm{group})$. ${ }^{\# \#} \mathrm{P}<0.01 \mathrm{vs}$. sham group; ${ }^{*} \mathrm{P}<0.05$ vs. TBI group. Scale bar, $50 \mu \mathrm{m}$. TBI, traumatic brain injury; Que, quercetin-treated.

A

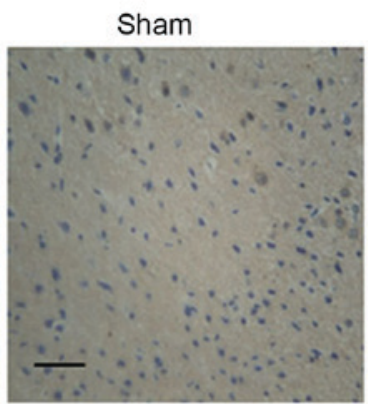

B

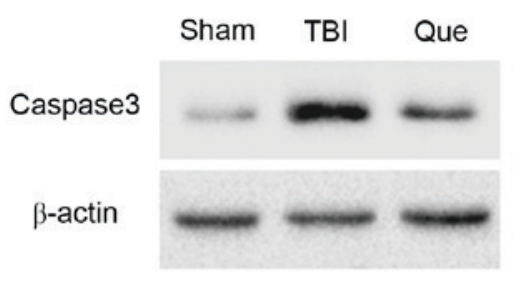

TBI

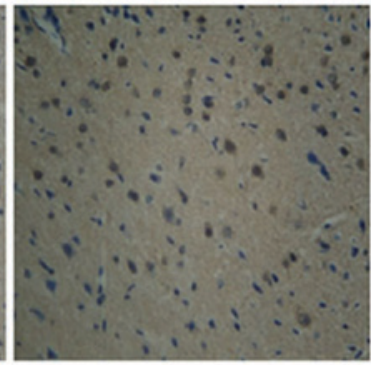

$\mathrm{C}$

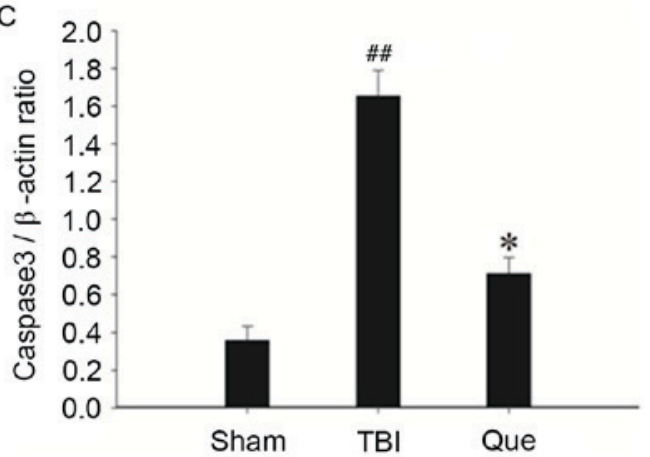

Figure 4. Effect of quercetin on Caspase 3 expression following TBI. (A) Representative images of immunohistochemical analysis of Caspase 3 in the sham, $\mathrm{TBI}$ and Que groups at $24 \mathrm{~h}$. Scale bar, $100 \mu \mathrm{m}$. (B) Western blot analysis demonstrating expression levels of caspase 3 in the cortex of rats at $24 \mathrm{~h}$. (C) The quantitative results of caspase 3 are expressed as the ratio of densitometries of caspase 3 to $\beta$-actin bands. Bars represent the mean \pm standard error of the mean ( $\mathrm{n}=5$ /group). The results demonstrated that the protein levels of caspase 3 increased significantly in the TBI group at $24 \mathrm{~h}\left({ }^{\# \#} \mathrm{P}<0.01 \mathrm{vs}\right.$. sham group), and the levels of caspase 3 exhibited significant downregulation at following treatment with quercetin ("P $<0.05$ vs. TBI group). TBI, traumatic brain injury; Que, quercetin-treated.

Quercetin induces the activation of the Akt signaling pathway in the cortex following TBI. Western blot analysis was performed to investigate the expression of Akt and p-Akt at
$24 \mathrm{~h}$ after TBI in the 3 groups. As presented in Fig. 5, the level of p-Akt was increased following TBI compared with that in the sham group $(\mathrm{P}<0.05)$. Additionally, administration of 


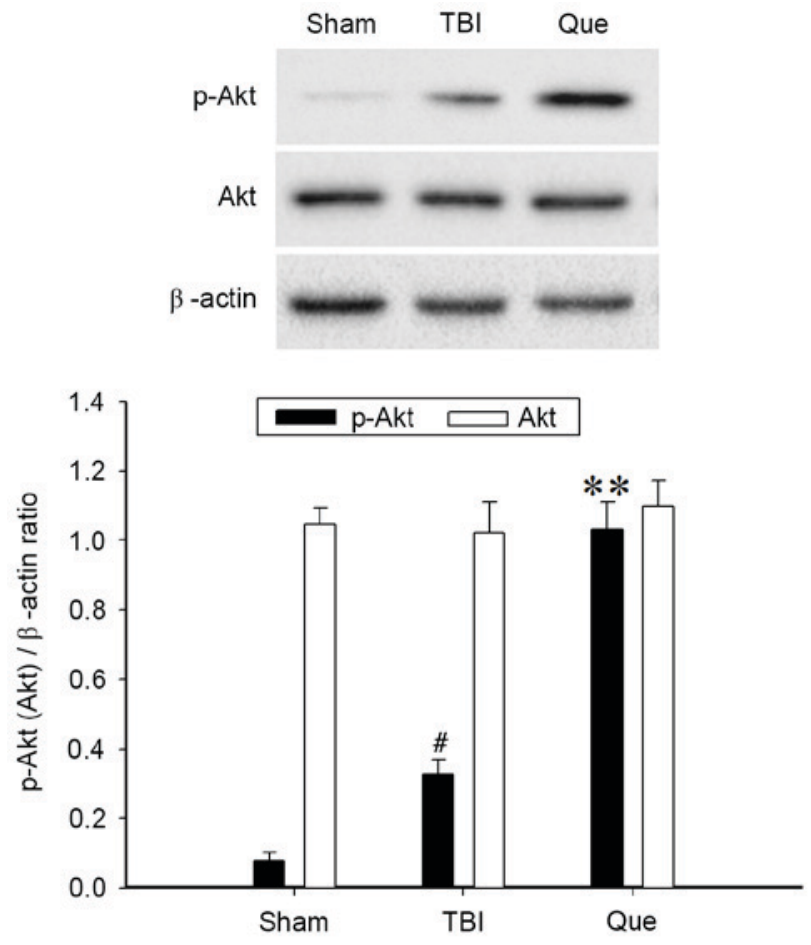

Figure 5. Effect of quercetin on the Akt pathway. Western blot analysis demonstrated the expression levels of Akt and p-Akt in the Sham, TBI and Que groups at $24 \mathrm{~h}$. The quantitative results of Akt and p-Akt are calculated as the ratio of the densitometries to $\beta$-actin bands, and expressed as the mean \pm standard error ( $\mathrm{n}=5 /$ group). ${ }^{*} \mathrm{P}<0.05$ vs. sham group; ${ }^{* *} \mathrm{P}<0.01$ vs. TBI group. TBI, traumatic brain injury; Que, quercetin-treated; Akt, Akt serine/threonine protein kinase; p, phosphorylated.

quercetin produced a significant elevation of p-Akt $(\mathrm{P}<0.01)$. No significant difference in total Akt protein expression was observed among the 3 groups.

Quercetin attenuates the ERK1/2 signaling pathway following $T B I$. Western blot analysis was performed to investigate the expression of ERK1/2 and p-ERK1/2 at $24 \mathrm{~h}$ after TBI in the 3 groups. As presented in Fig. 6, the level of p-ERK1/2 was increased significantly post-TBI, compared with the sham group $(\mathrm{P}<0.01)$. However, the administration of quercetin produced a significant attenuation of $\mathrm{p}$-ERK1/2 levels compared with the TBI group $(\mathrm{P}<0.01)$. No significant difference in total ERK1/2 protein expression was observed among the 3 groups.

\section{Discussion}

The aim of the present study was to investigate the neuroprotective effects of quercetin on TBI. H\&E staining is a macroscopic and readily available method to assess histopathological changes. Quercetin treatment notably attenuated injury. In the Que group, the structure of the brain tissue was improved and the number of neurons increased compared with the TBI group. In addition, TBI-induced neurological deficits and brain edema was suppressed by treatment with quercetin. At the molecular level, treatment with quercetin significantly inhibited the TBI-induced expression of cleaved Caspase 3 . It was additionally observed that the neuroprotective effects of the drug were associated

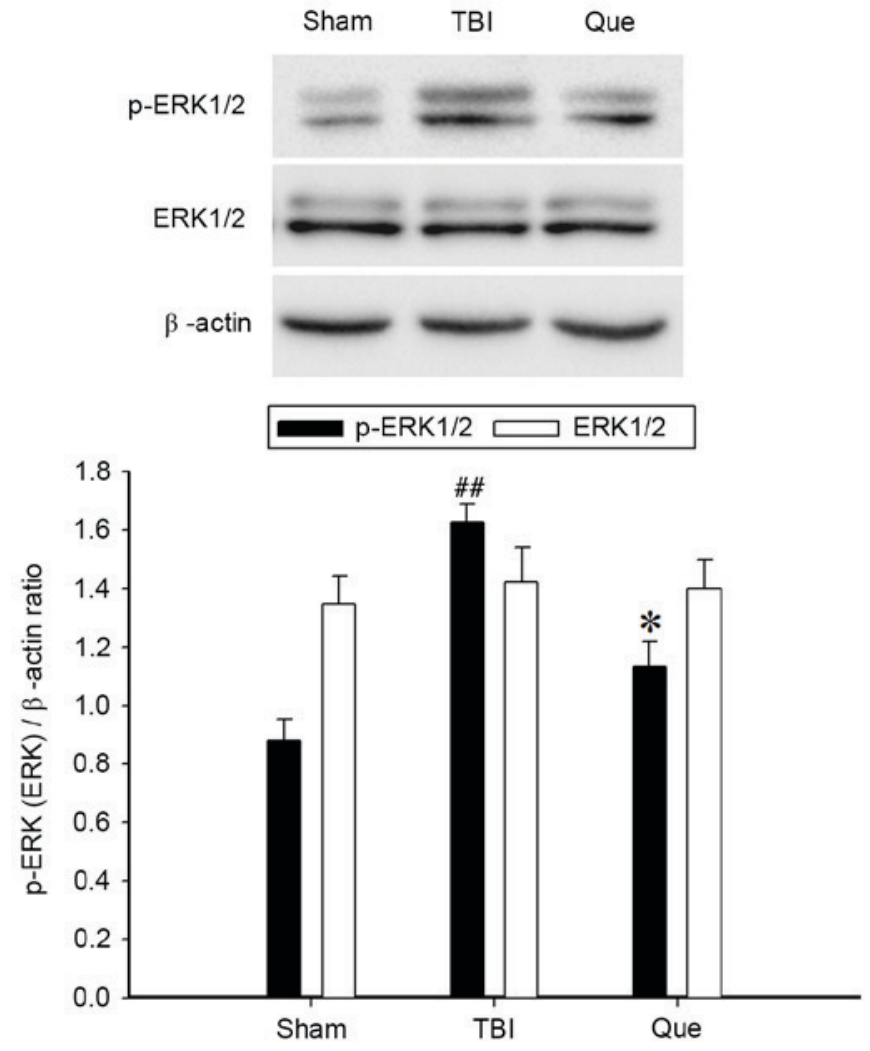

Figure 6. Effect of quercetin on the ERK1/2 signaling pathway. Western blot analysis demonstrated the expression levels of ERK1/2 and p-ERK1/2 in the sham, TBI and Que groups at $24 \mathrm{~h}$. The quantitative results of ERK1/2 and p-ERK $1 / 2$ are calculated as the ratio of the densitometries to $\beta$-actin bands, and expressed as the mean \pm standard error ( $\mathrm{n}=5$ /group). ${ }^{\# \#} \mathrm{P}<0.01$ vs. sham group; ${ }^{* *} \mathrm{P}<0.01$ vs. TBI group. TBI, traumatic brain injury; Que, quercetin-treated; ERK, extracellular signal-regulated kinase; p, phosphorylated.

with activation of the Akt signaling pathway, and inhibition of the ERK signaling pathway. The results of the present study were consistent with previous studies demonstrating that quercetin may exert neuroprotection in various in vitro and in vivo models (12-15). Therefore, it is hypothesized that quercetin may have the potential to become a novel therapeutic for TBI.

The primary injury occurs at the moment of TBI impact, with disruption of the blood brain barrier and blood vessels that contribute to immediate (necrotic) cell death (16). Subsequently, oxygen free radical-mediated lipid peroxidation, inflammation and brain edema appear to be fundamental mechanisms underlying secondary damage in TBI (17). In the present study, caspase 3 was induced by TBI, which is a key executor in the process of apoptosis in neurons (18). However, treatment with quercetin significantly inhibited the TBI-induced expression of cleaved caspase 3 . These observations were consistent with a previous study, which demonstrated that caspase 3 immunoreactivity was reduced by quercetin in the cerebral ischemic penumbra in rats (19). The neuroprotective effect of quercetin was associated with the inhibition of neuronal apoptosis.

Akt, also termed protein kinase $\mathrm{B}$, is a serine/threonine kinase with pro-survival functions in acute brain injury (20). Extracellular signals frequently result in the simultaneous activation of the PI3K/Akt signaling 
pathway, and a number of reports have suggested a survival role of the PI3K/Akt signaling pathway through the suppression of apoptosis $(21,22)$. Additionally, previous studies have demonstrated that the intrinsic pathway is characterized by mitochondrial outer membrane permeabilization, death-inducible signaling complex formation, DNA fragmentation and caspase 3 activation. These events have been demonstrated to be associated with ERK1/2 signaling pathway activation $(23,24)$. In the present study, treatment with quercetin inhibited the TBI-induced activation of the ERK1/2 signaling pathway, and further enhanced the PI3K/Akt pathway in TBI-injured neurons. Therefore, the neuroprotective effects of quercetin may be associated with ERK1/2 inhibition in addition to PI3K/Akt activation.

Previous studies have showed that in TBI rats, quercetin improves cognitive function due to its neuroprotective action via the inhibition of oxidative stress, leading to a reduced inflammatory response and thereby reducing neuronal death $(7,8)$. Compared with other studies, the present study performed a more in-depth study on the molecular mechanism of the neuroprotection effects of quercetin. It was demonstrated that post-TBI administration of quercetin may attenuate brain edema and improve motor functions in rats. In addition, quercetin caused marked ERK1/2 inhibition and PI3K/Akt activation, and thereby attenuation of neuronal apoptosis. The present study provides novel insight into the mechanisms through which quercetin may exert its neuroprotective activity in a rat model of TBI.

\section{Acknowledgements}

Not applicable.

\section{Funding}

No funding was received.

\section{Availability of data and materials}

All datasets on which the conclusions are based are provided in the present article.

\section{Authors' contributions}

GD, ZZ and YC designed the present study. ZoL, YT and ZhL performed the experiments. BL and JS analyzed and interpreted data, and were major contributors in writing the manuscript. All authors read and approved the final manuscript.

\section{Ethics approval and consent to participate}

The Institutional Animal Care and Use Committee of Hebei Medical University (Shijiazhuang, China) approved all experiments, which were performed according to the guidelines of the National Institutes of Health (NIH) Guide for the Care and Use of Laboratory Animals (NIH Publications no. 80-23, revised 1978; NIH, Bethesda, MD, USA). All efforts were made to minimize the number of animals used and their suffering.

\section{Consent for publication}

Not applicable.

\section{Competing interests}

The authors declare that they have no competing interests.

\section{References}

1. Ye X, Asim M and Michael C: Animal models of traumatic brain injury. Nat Rev Neurosci 14: 128-142, 2013.

2. Carroll LJ, Cassidy JD, Cancelliere C, Côté P, Hincapié CA Kristman VL, Holm LW, Borg J, Nygren-de Boussard C and Hartvigsen J: Systematic review of the prognosis after mild traumatic brain injury in adults: Cognitive, psychiatric, and mortality outcomes: Results of the international collaboration on mild traumatic brain injury prognosis. Arch Phys Med Rehabil 95 (3 Suppl): S152-S173, 2014.

3. Verma AK and Pratap R: The biological potential of flavones. Nat Prod Rep 27: 1571-1593, 2010.

4. Legault J, Perron T, Mshvildadze V, Girard-Lalancette K, Perron S, Laprise C, Sirois P and Pichette A: Antioxidant and anti-inflammatory activities of quercetin 7-O- $\beta$-D-glucopyranoside from the leaves of Brasenia schreberi. J Med Food 14: 1127-1134, 2011.

5. Zhang H, Zhang M, Yu L, Zhao Y, He N and Yang X: Antitumor activities of quercetin and quercetin-5',8-disulfonate in human colon and breast cancer cell lines. Food Chem Toxicol 50: 1589-1599, 2012

6. Dok-Go H, Lee KH, Kim HJ, Lee EH, Lee J, Song YS, Lee YH, Jin C, Lee YS and Cho J: Neuroprotective effects of antioxidative flavonoids, quercetin, (+)-dihydroquercetin and quercetin 3-methyl ether, isolated from Opuntia ficus-indica var. saboten. Brain Res 965: 130-136, 2003.

7. Yang T, Kong B, Gu JW, Kuang YQ, Cheng L, Yang WT, Xia X and Shu HF: Anti-apoptotic and anti-oxidative roles of quercetin after traumatic brain injury. Cell Mol Neurobiol 34: 797-804, 2014.

8. Du G, Zhao Z, Chen Y, Li Z, Tian Y, Liu Z, Liu B and Song J: Quercetin attenuates neuronal autophagy and apoptosis in rat traumatic brain injury model via activation of PI3K/Akt signaling pathway. Neurol Res: 1-8, 2016 (Epub ahead of print).

9. Marmarou A, Foda AE, van den Brink W, Campbell J, Kita H and Demetriadou K: A new model of diffuse brain injury in rats. Part I: Pathophysiology and biomechanics. J Neurosurg 80: 291-300, 1994.

10. Chen Y, Constantini S, Trembovler V, Weinstock M and Shohami E: An experimental model of closed head injury in mice: Pathophysiology, histopathology, and cognitive deficits. J Neurotrauma 13: 557-568, 1996.

11. Donkin JJ and Vink R: Mechanisms of cerebral edema in traumatic brain injury: Therapeutic developments. Curr Opin Neurol 23: 293-299, 2010.

12. Zhang ZJ, Cheang LC, Wang MW and Lee SM: Quercetin exerts a neuroprotective effect through inhibition of the iNOS/NO system and pro-inflammation gene expression in PC12 cells and in zebrafish. Int J Mol Med 27: 195-203, 2011.

13. Kumar B, Gupta SK, Nag TC, Srivastava S, Saxena R, Jha KA and Srinivasan BP: Retinal neuroprotective effects of quercetin in streptozotocin-induced diabetic rats. Exp Eye Res 125: 193-202, 2014.

14. Pu F, Mishima K, Irie K, Motohashi K, Tanaka Y, Orito K, Egawa T, Kitamura Y, Egashira N, Iwasaki K and Fujiwara M: Neuroprotective effects of quercetin and rutin on spatial memory impairment in an 8-arm radial maze task and neuronal death induced by repeated cerebral ischemia in rats. J Pharmacol Sci 104: 329-334, 2007.

15. Silva B, Oliveira PJ, Dias A and Malva JO: Quercetin, kaempferol and biapigenin from Hypericum perforatum are neuroprotective against excitotoxic insults. Neurotox Res 13: 265-279, 2008.

16. Eucker SA, Smith C, Ralston J, Friess SH and Margulies SS: Physiological and histopathological responses following closed rotational head injury depend on direction of head motion. Exp Neurol 227: 79-88, 2011. 
17. Feng JF, Gurkoff GG, Van KC, Song M, Lowe DA, Zhou J and Lyeth BG: NAAG peptidase inhibitor reduces cellular damage in a model of TBI with secondary hypoxia. Brain Res 1469: 144-152, 2012.

18. Clark RS, Kochanek PM, Watkins SC, Chen M, Dixon CE Seidberg NA, Melick J, Loeffert JE, Nathaniel PD, Jin KL and Graham SH: Caspase-3 mediated neuronal death after traumatic brain injury in rats. J Neurochem 74: 740-753, 2000.

19. Yao RQ, Qi DS, Yu HL, Liu J, Yang LH and Wu XX: Quercetin attenuates cell apoptosis in focal cerebral ischemia rat brain via activation of BDNF-TrkB-PI3K/Akt signaling pathway. Neurochem Res 37: 2777-2786, 2012.

20. Endo H, Nito C, Kamada H, Yu F and Chan PH: Akt/GSK3beta survival signaling is involved in acute brain injury after subarachnoid hemorrhage in rats. Stroke 37: 2140-2146, 2006.

21. Park KR, Nam D, Yun HM, Lee SG, Jang HJ, Sethi G, Cho SK and Ahn KS: $\beta$-Caryophyllene oxide inhibits growth and induces apoptosis through the suppression of PI3K/AKT/mTOR/S6K1 pathways and ROS-mediated MAPKs activation. Cancer Lett 312: $178-188,2011$.
22. Li WX, Chen SF, Chen LP, Yang GY, Li JT, Liu HZ and Zhu W: Thimerosal-induced apoptosis in mouse C2C12 myoblast cells occurs through suppression of the PI3K/Akt/survivin pathway. PLoS One 7: e49064, 2012.

23. Yan L, Tang Q, Shen D, Peng S, Zheng Q, Guo H, Jiang M and Deng W: SOCS-1 inhibits TNF-alpha-induced cardiomyocyte apoptosis via ERK1/2 pathway activation. Inflammation 31: 180-188, 2008

24. Chong YH, Shin YJ, Lee EO, Kayed R, Glabe CG and Tenner AJ: ERK1/2 activation mediates Abeta oligomer-induced neurotoxicity via caspase-3 activation and tau cleavage in rat organotypic hippocampal slice cultures. J Biol Chem 281: 20315-20325, 2006.

This work is licensed under a Creative Commons

Attribution-NonCommercial-NoDerivatives 4.0 International (CC BY-NC-ND 4.0) License. 